\title{
Comparative Study on Various Edge Detection Techniques for 2-D Image
}

\author{
Kamlesh Kumar \\ School of Computer Science \& \\ Engineering \\ UESTC, Chengdu, 611731, \\ China
}

\author{
Jian- Ping Li \\ School of Computer Science \& \\ Engineering \\ UESTC, Chengdu, 611731, \\ China
}

\author{
Saeed Ahmed Khan \\ Department of Electrical \\ Engineering, Sukkur Institute \\ of Business Administration, \\ Sindh, Pakistan
}

\begin{abstract}
This research work is extended version of our previous work, which was carried out on 2-D image edge detection using daubachies $\mathrm{db} 10$ wavelet transform. Here in this paper, the $\mathrm{db} 10$ wavelet transform for edge detection is compared with most widely used edge detection techniques, such as Sobel, Prewitt, Roberts and Laplacian of Gaussian (LoG) and Canny. The comprehensive studies along with pros and cons of each edge method have been explained. 2-D image of different edge detection techniques have been generated in MATLAB and results are being compared.
\end{abstract}

\section{General Terms}

Edge detection, Daubachies db10, Sobel, Prewitt, Roberts and Laplacian of Gaussian (LoG) and Canny

\section{Keywords}

Wavelet Transforms; Daubachies; Edge Detection

\section{INTRODUCTION}

Edge detection has significant importance in the field of image processing and computer vision, which is used for feature matching, object recognition and segmentation. Edge detection is one kind of segmentation technique [1], which bisects an image into well coherent or incoherent regions so that intended scenes may be captured. It is needed in many applications for delineating the specific objects, such as in biomedical images, human-computer interaction, surveillance $\&$ monitoring and content based information retrieval. There are various edge detection techniques [2], which have been used by different researchers for finding the edges in the image. The most widely used techniques are Sobel, Prewitt, Roberts, Log and Canny, while each of them suffers from some shortcomings and produces errors in the advent of locating edges. Here in this paper, comprehensive study has been done on different edge finding methods and also compared with wavelet based edge detection technique [3]. The results are evaluated on carrying 2-D intensity image.

\subsection{Edge and Its Techniques}

Edge is defined as abrupt changes or discontinuities in the intensity of image, which is used for highlighting and locating the boundaries of the image's object. The main advantage of using edge detection is that it reduces the amount of data without considerable loss and preserves high level information of the object such as its shape for further processing [4]. However, good edge detection depends upon the edge filter. The chosen filter must be invariant to translation, rotation and scale of the image, while finding the edge. The edge detection techniques fall into two main categories [5], one is gradient method and other is laplacian method. Former one finds edge by gradient maxima and minima Intensity ' $I$ ' in the first derivative of the image and later one finds zero crossing in the second derivative of the image.

\section{DIFFERENT EDGE DETECTION METHODS}

\section{Sobel Edge Method}

Sobel edge method uses a filter that convolves an input image with a kernel and calculates the gradient magnitude and direction of each image pixel. The magnitude of the vector is expressed as:

$$
\Delta f=\operatorname{mag}(\Delta f)=\left[G_{x}^{2}+G_{y}^{2}\right]^{\frac{1}{2}}
$$

The $G_{x}$ and $G_{y}$ in the equation represent horizontal and vertical direction. The sobel uses two pairs of $3 \times 3$ kernels for $\mathrm{x}$ and $\mathrm{y}$ direction.

$$
\begin{array}{ll}
\mathrm{x} \text { - direction } & \mathrm{y} \text {-direction } \\
{\left[\begin{array}{ccc}
-1 & -2 & -1 \\
0 & 0 & 0 \\
1 & 2 & 1
\end{array}\right]} & {\left[\begin{array}{ccc}
-1 & 0 & 1 \\
-2 & 0 & 2 \\
-1 & 0 & 1
\end{array}\right]}
\end{array}
$$

Fig 1: Sobel 3x3 Kernels

One kernel is simply the other rotated by $90^{\circ}$.

\section{Prewitt Edge Method}

Prewitt edge method resembles with sobel edge method. It belongs to first derivative, which detects edges along horizontal $G_{x}$ and vertical $G_{y}$ direction and calculates the difference of image pixel intensities on edge region.

The prewitt kernels for $G_{x}$ and $G_{y}$ are defined as:

$$
\begin{array}{cc}
\text { X-direction } & \text { y-direction } \\
{\left[\begin{array}{ccc}
-1 & -1 & -1 \\
0 & 0 & 0 \\
1 & 1 & 1
\end{array}\right]} & {\left[\begin{array}{ccc}
-1 & 0 & 1 \\
-1 & 0 & 1 \\
-1 & 0 & 1
\end{array}\right]}
\end{array}
$$

Fig 2: Prewitt 3x3 Kernels

Here in Fig 2: it shows that in $\mathrm{x}$ and $\mathrm{y}$ direction of two $3 \times 3$ matrix, central elements are containing 0 , so they will not be included in the original values of edge in the image. It calculates the difference of pixel intensities along $x$ - direction for above and below pixel values and also along y- direction, it will calculate right and left pixel values for edge and thereby increases the edge intensity. 


\section{Roberts Edge Method}

Roberts's edge method is considered as simple and efficient differential operator. This approximates the gradient intensity in discrete differentiation, which is obtained by summation of squares of differences between diagonally adjacent pixels.

It uses a pair of $2 \times 2$ convoluted kernels, which is like sobel edge method.

\begin{tabular}{|r|r|}
\hline+1 & 0 \\
\hline 0 & 1 \\
\hline
\end{tabular}

Gx

\begin{tabular}{|c|c|}
\hline 0 & +1 \\
\hline-1 & 0 \\
\hline
\end{tabular}

Gy
Fig 3: Roberts $x, y$ kernels

The both kernels have been designed in such a way that that response maximally to edges lying at $45^{\circ}$ angle in the pixel grid. It holds one kernel for each two perpendicular orientations. However kernels can be applied individually to input image, which produce separated measurements of the gradient in $\mathbf{G}_{\mathbf{x}}$ and $\mathbf{G}_{\mathbf{y}}$ orientation. Each one is combined together to find absolute magnitude of gradient at individual point and orientation. The gradient magnitude is given as:

$$
|G|=\sqrt{G x^{2}}+\sqrt{G y^{2}}
$$

Approximate magnitude as well as angle of orientation of the edge that give rise to the spatial gradient is also computed in equation: $3 \& 4$ :

$$
\begin{gathered}
|G|=\left|G_{x}\right|+\left|G_{y}\right| \\
\theta=\arctan \left(\frac{G_{x}}{G_{y}}\right)-3 \pi / 4
\end{gathered}
$$

\section{Laplacian of Gaussian (LoG) Edge Method}

The Laplacian of Gaussian (LoG) is also called MarrHilderath edge method, which belongs to second spatial derivative of an image. This method requires pre-processing step, an image $\mathrm{f}(\mathrm{x}, \mathrm{y})$ is smoothed with guassian filter in order to get rid of the noise sensitivity issue and later on combined with laplacian filter, which detects the true edges effectively. The edges are analysed by looking at zero crossing after smoothed with laplacian of guassian filter. The filter usually takes grey level input image and produce grey level image as output. The Laplacian $(x, y)$ of an image with pixel intensity values $I(x, y)$ is given as:

$$
L(x, y)=\frac{\partial^{2} I}{\partial x^{2}}+\frac{\partial^{2} I}{\partial y^{2}}
$$

The 2-D Gaussian function is explained as:

$$
h(x, y)=-e \frac{x^{2}+y^{2}}{2 \sigma^{2}}
$$

Here sigma $\sigma$ is the standard deviation, which blurs the image and blurring is determined by sigma value.

\section{Canny Edge Method}

The canny edge method performs multi edge detection technique, while finding the image edges. At initial level, it applies a guassian filter that smooths the image so as to wipe out noise.
Gaussian filter kernel with the size of $2 \mathrm{k}+1 * 2 \mathrm{k}+1$ is defined as:

$$
H i j=\frac{1}{2 \pi \sigma^{2}} * \exp \left(-{\frac{(i-k-1)^{2}+(j-k-1)^{2}}{2 \sigma^{2}}}^{2}\right)
$$

Then, it finds the image gradient for high intensities pixels and suppresses the low intensity pixels (non-maximum suppression). The edge gradient and direction can be determined by equation: (8) \& (9) as given below:

$$
\begin{aligned}
G & =\sqrt{G x^{2}+G y^{2}} \\
\Theta & =a \tan 2(G y, G x)
\end{aligned}
$$

In the end it uses hysteresis operation, to eliminate pixels that have not been suppressed. Two Thresholds, T1 and T2 is selected for hysteresis, which checks if the magnitude is below the level T1, set to zero and mark it as non-edge. If the magnitude is above the level of T2, set to one and mark it as edge. Meanwhile, if the magnitude is in the middle between the $\mathrm{T} 1$ and $\mathrm{T} 2$ then make it zero unless there is a path from this pixel to another pixel with a gradient above $\mathrm{T} 2$.

\section{PROPOSED APPROACH}

Here, comparative studies for 2-D image edge detection have been carried by approaching different edge detection methods [6]. MATLAB code for different edge methods is generated for experimental evaluation of results. However, wavelet transform is main focus for this research work. For this two algorithms have been generated for image decomposition and reconstructions of 2-D image by implementing (Db10) wavelet transform [7].

An edge is a boundary between two regions, which means variation of intensity level will cause an edge. We have to find out location, where intensity changes rapidly between two different shades, edge will be detected. Edges are actually high frequencies in an image, therefore, there is need to use some sort of filter, which separates higher frequencies and lower frequencies. The advantage of using wavelet transform [8] is that it gives out higher and lower frequencies separately, so it is easy to get the edges with this technique. Moreover, the cut-off frequencies of wavelet filters are variable which results in good time and frequency resolution.

\subsection{Wavelet and Its Different Schemes}

Wavelet is useful mathematical tool for signal analysis that has gained remarkable achievements in digital signal processing as well as image compression [9]. It provides a mechanism to visualize the non- stationary signal by taking time and frequency information simultaneously [10]. Wavelet consists of a basic function $\psi(x)$ called as "mother wavelet, and $\psi^{a, b}(x)$ which is confronted in a finite interval and from that "Daughter wavelets" can be constructed through translation and contraction. The main advantage of wavelet is that the signal can be viewed at different scales by analyzing low and high frequency components. Wavelets are categorized into different families according to their functionality, which have been explained in our earlier paper work [11].

\section{METHODOLOGY OF EDGE DETECTION METHOD}

Here the 2-D image is decomposed by Db10 wavelet and then reconstructed for edge detection. In fig 4: an original image is shown below and algorithm is applied on it. 


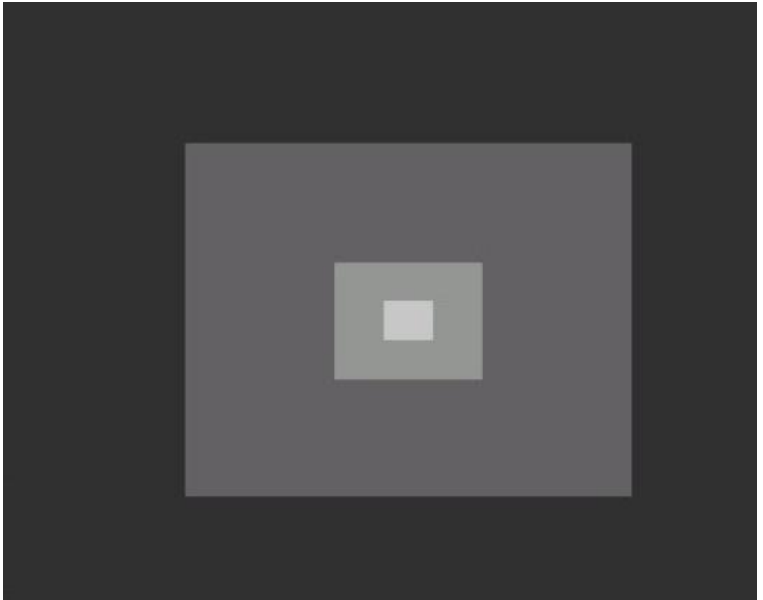

Fig 4: Original Image

\section{ALGORITHM FOR EDGE DETECTION}

1. Load an image.

2. Apply low and high pass filter.

3. As data has got doubled so down sample it (discrete filters).

4. Create an array and save them.

5. Now, the result will show approximations as well as details components for lower and higher frequencies.

6. This will show first level decomposition.

7. These coefficients will be saved in a workspace.

8. Repeat steps from 2 to 7 by taking first level decomposed approximation and details coefficients

9. This process is repeated till an appropriate level.

10. Our approximations represent lower frequencies and details contain higher frequencies.

11. However we have three types of details, horizontal, vertical and diagonal.

12. These horizontal, vertical and diagonal details will represent edges respectively.

13. Finally decomposed image will be displayed.

In fig 5: the decomposed image is shown, when algorithm is run in the MATLAB.

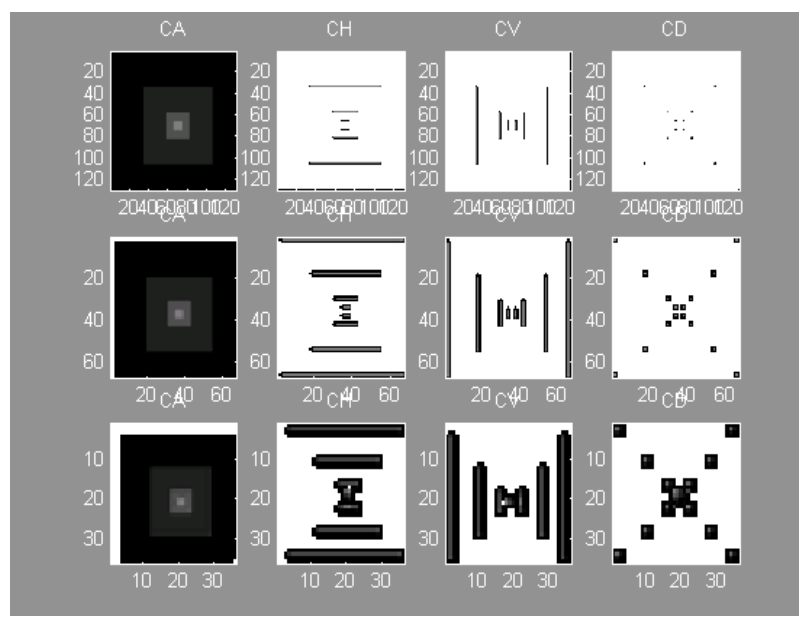

Fig 5: Decomposed Image

The original image has been decomposed at three levels. The left most CA1, CA2, CA3 represents approximates coefficient for lower frequencies, while next to them are horizontal $(\mathrm{CH} 1$, $\mathrm{CH} 2, \mathrm{CH} 3$ ), vertical (CV1, CV2, CV3), and diagonal (DC1,
DC2, DC3), which represents details co-efficient for higher frequencies. Actually details are edges, so we will suppress the approximations effect to zero. If the edges are not obvious then thresholding is needed to cut the ghost edges.

An algorithm for image reconstruction for the sake of image edge detection is devised below.

\section{ALGORITHM FOR IMAGE RECONSTRUCTION}

1. Take approximations and details coefficients for three level decomposition.

2. Apply up sampling.

3. Apply low and high pass filter (Reconstructed filter).

4. Create an array and save them.

5. Combine them and get approximations and details.

6. Save them in workspace.

7. Now, this will be first level reconstruction.

8. Repeat steps from 2 to 6 by taking first level reconstructed approximations and details.

9. This process is iterated until we get original image.

10. Here, we will get an edge of original image at $\mathrm{CA} 3=0$

11. Meanwhile, apply thresholding to suppress ghost edges.

12. Finally display the edges of the image.

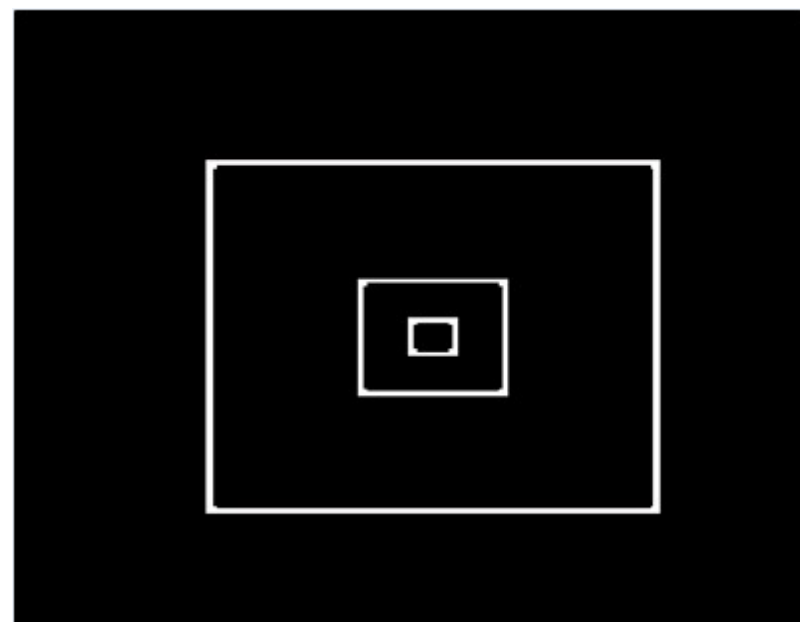

Fig 6: Edges of the Image

It is worthwhile to see in the fig 6: wavelet reconstructed image have detected sharp edges. However, Daubechies-10 wavelet gives appropriate edges for the 2-D image at three levels. In case of ghost edges, thresholding is also used to nullify them.

\section{EDGE DETECTION EXPRIMENTAL RESULTS AND DISCUSSION}

The 2-D image edge detection has been performed on different edge detection methods. However comparative analyses have been done by experimental results. The Fig 7: below depicts an original image which has been used as a template for edge detection. The fig 8: to fig 13: represents edge detected images respectively. It can been observed from these images that Db10 wavelet has detected very sharp and bright edges, while Sobel and Prewitt method has almost similar output but their edge corners looks like curlier. The Roberts as well as Canny method has produced visible edges beside some edge line discontinuity. In the last Laplacian of guassian method has located edge points, which are thinner and seems not clear. 


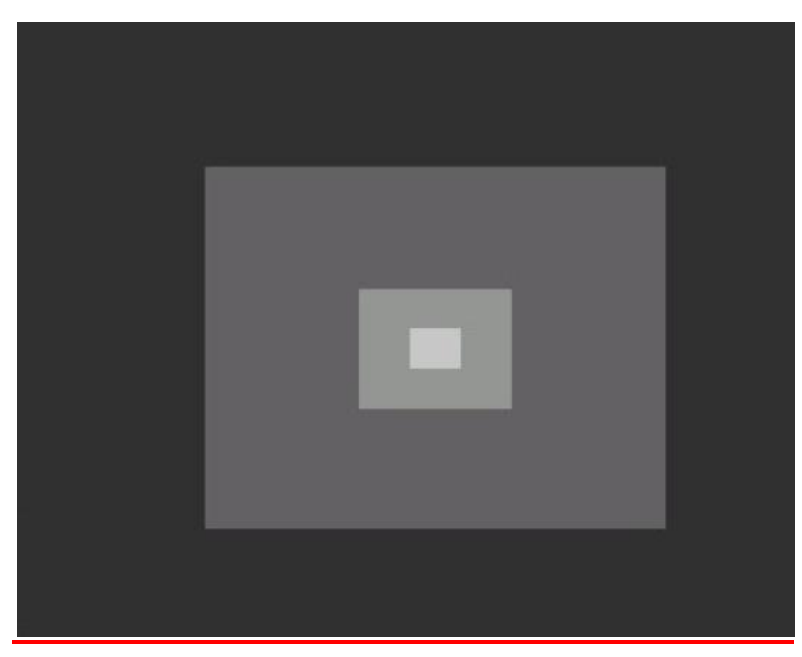

Fig 7: Original Image

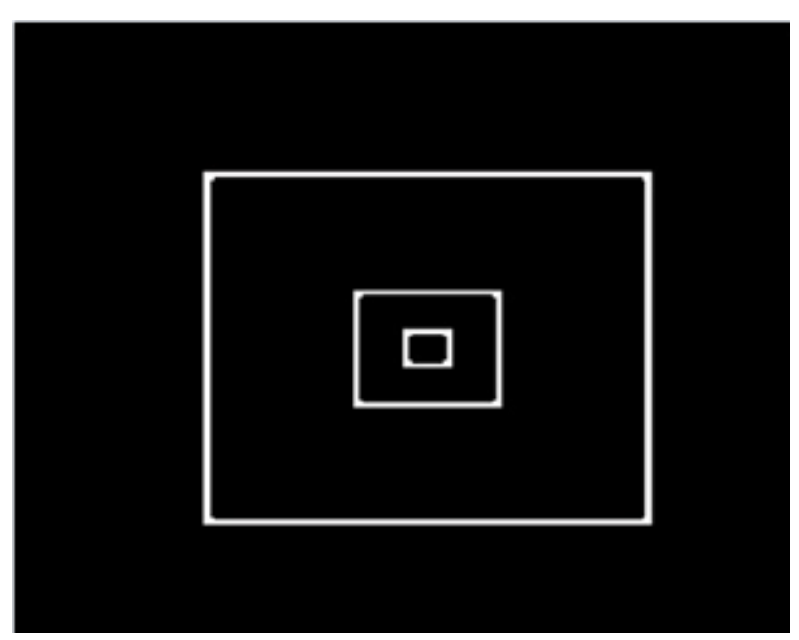

Fig 8: Wavelet Db10 Edge Detection Method

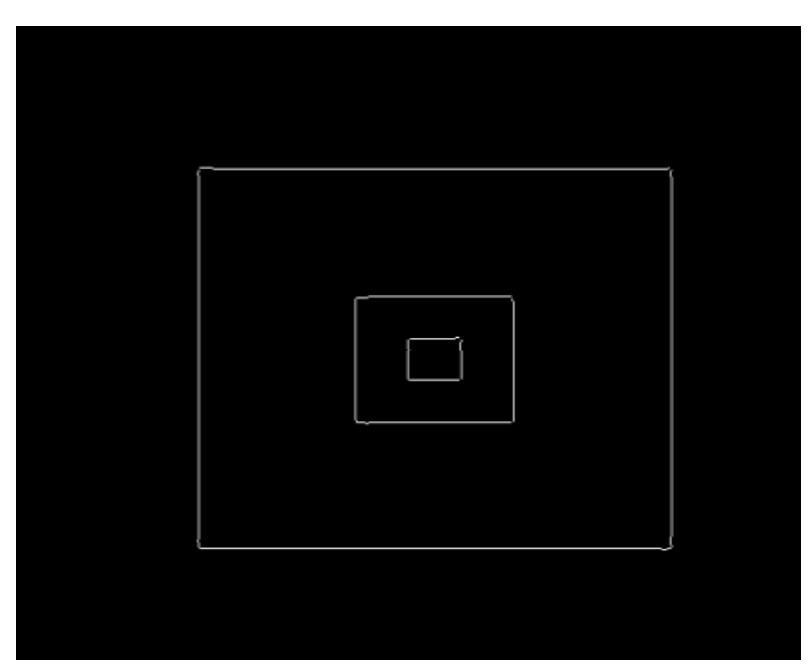

Fig 9: Sobel Edge Detection Method

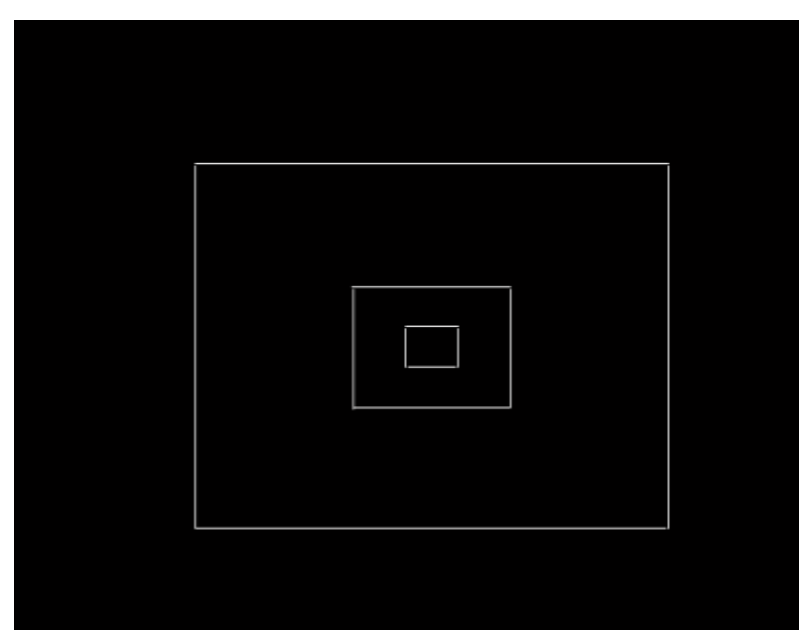

Fig 10: Prewitt Edge Detection Method

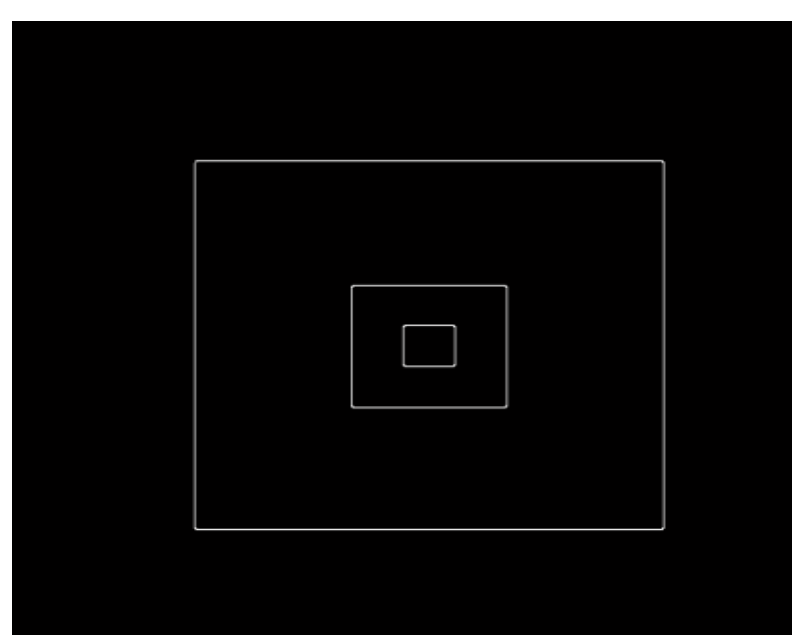

Fig 11: Roberts Edge Detection Method

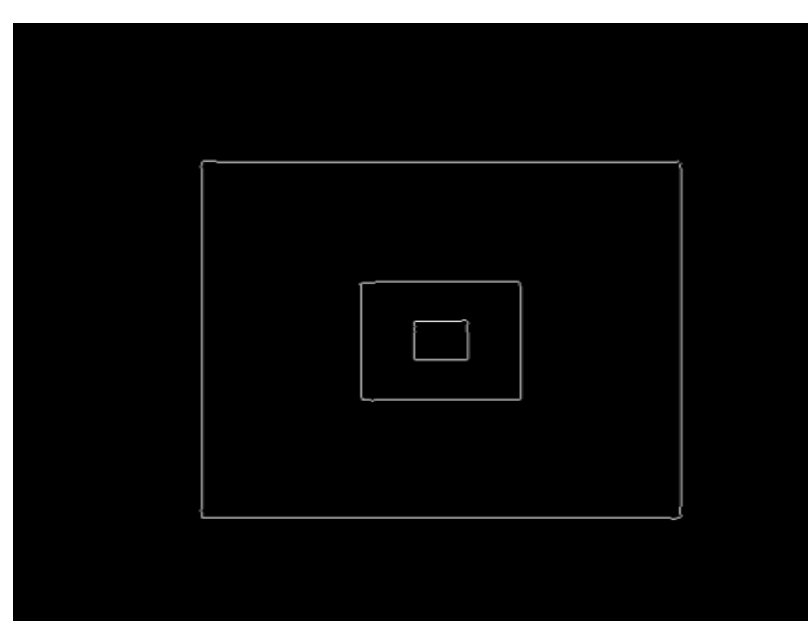

Fig 12: Canny Edge Detection Method 


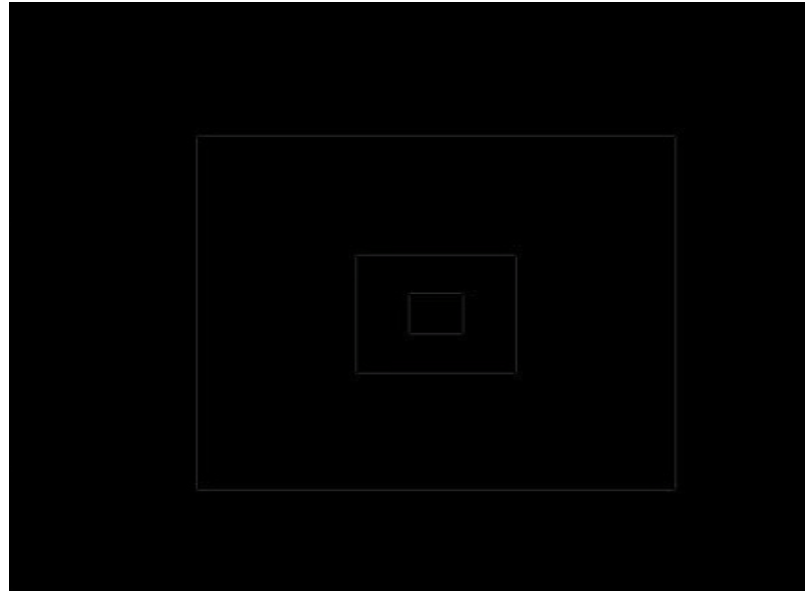

Fig 13: LOG Edge Detection Method

\subsection{Pros and Cons of Different Edge Methods}

1. The first derivative gradient based edge methods such as Sobel, Prewitt and Roberts are simple in computation with good edge orientation detection but these does not produce sharp edges and their performance is reduced for noisy images.

2. The second derivative laplacian of guassian method is good at finding correct location of edges along a large pixel area, but produces false edges due to high intensity change at corners and curves with poor edge orientation.

3. Canny edge is an optimal method amongst those techniques. It provides better response over edge location especially when images are corrupted with noise. This uses two thresholds one for weak edges and other for strong edges which is time consuming. However it suffers from false zero crossing, which results in missed true edges.

4. Wavelet analyzes signals through low pass and high pass filter, which has been used successfully for image edge detection. It provides local view of the signal at different scales and meanwhile separates high frequencies for edges and low frequencies for unwanted information. The drawback of this method is that it requires appropriate decomposition level for image, if it is not considered; the noise will be added to true edges.

\section{CONCLUSION}

In this paper comprehensive study on edge detection techniques was evaluated for 2-D image. The experimental results showed that, our db10 wavelet technique achieved high response in edge detection while comparison with all investigated edge detection methods. This method is simple as well as efficient for 2-D images. The edge detection is crucial for object detection in computer vision and image processing. In our future work, the algorithm will be realized for object recognition and retrieval.

\section{ACKNOWLEDGEMENT}

This paper was supported by the National Natural Science Foundation of China Grant No.61370073 and National HighTech R\&D Program of China Grant No.2007AA01Z423.

\section{REFERENCES}

[1] N. Senthilkumaran and R. Rajesh. "An Edge Detection Techniques for Image Segmentation", International Journal of Recent Trends in Engineering, Vol. 1(2), pp. 250-255, May.2009.

[2] Rashmi, Mukesh Kumar. And Rohini, "Algorithm and Techniques on Various Edge Detection", Signal and Image Processing Journal, Vol.4 (3), pp. 56-75, June. 20013.

[3] E. Brannock and M. Weeks, "Edge detection using wavelets", ACM SE 06, Melbourne, Florida, USA. ACM (March 2006,M. Wegmuller, J. P. von der Weid, P. Oberson, and N. Gisin, "High resolution fiber distributed measurements with coherent OFDR," in Proc. ECOC'00, 2000, paper 11.3.4, p. 109.

[4] Mohamed Abo-Zahhad, Reda Ragab Ghrieb, "Edge Detection with a Preprocessing Approach", Journal of Signal and Information Processing, pp. 123-134, November. 2014.

[5] Wang Luo, "Comparison for Edge Detection of Colony Images", International Journal of Computer Science and Network Security, Vol.6 (9A), pp. 211-215, September. 2006.

[6] Jun li, "A Wavelet Approach to Edge Detection." Thesis (Masters), Sam Houston State University, Huntsville, Texas, August. 2003.

[7] DAUBECHIES, Ingrid. Ten Lectures on Wavelets, Philadelphia, Pennsylvania, Society for Industrial and Applied Mathematics, 1992. CBMS-NSF regional conference series in applied mathematics; vol. 61. ISBN 0898712742 .

[8] Li Wan-She, Zhao Jin, “ Application of Wavelet Transform in Edge Detection", Image and Signal Processing Conference, Vol. 4, pp. 2173-2176, October. 2011.

[9] Polikar99, Robi Polikar, "The Wavelet Tutorial", An Overview of Wavelet Theory, Aug1999.

[10] J. J. Ding, "Time Frequency Analysis and Wavelet Transform", http://disp.ee.ntu.edu.tw/tutorial.php.

[11] Kamlesh Kumar, Jian-Ping Li, Saeed Ahmed Khan, "Image Edge Detection Scheme Using Wavelet Transform", International Computer Conference on Wavelet Active Media Technology and Information Processing, Vol. 11, pp. 261-265, December. 2014 